\title{
SOME REMARKS ON EXTREMAL PROBLEMS IN WEIGHTED BERGMAN SPACES OF ANALYTIC FUNCTIONS
}

\author{
ROMI F. SHAMOYAN AND MILOŠ ARSENOVIĆ $\dagger$
}

\begin{abstract}
We prove some sharp extremal distance results for functions in weighted Bergman spaces on the upper halfplane. We also prove new analogous results in the context of bounded strictly pseudoconvex domains with smooth boundary.
\end{abstract}

\section{INTRODUCTION}

If $Y$ is a normed space and $X \subset Y$, then we set $\operatorname{dist}_{Y}(f, X)=\inf _{g \in X}\|f-g\|_{Y}$. If the space $Y$ is clear from the context, we write $\operatorname{simply} \operatorname{dist}(f, X)$. In the problems we are going to consider, $X$ itself is going to be a (quasi)-Banach space.

We denote by $H(\Omega)$ the space of all holomorphic functions on an open set $\Omega \subset$ $\mathbb{C}^{n}$. In this paper we consider distance problems in weighted Bergman spaces over the upper half-plane $\mathbb{C}_{+}=\{x+i y: y>0\}$ and over a bounded strictly pseudoconvex domain $\Omega \subset \mathbb{C}^{n}$ with smooth boundary.

The weighted Bergman space $A_{\alpha}^{p}\left(\mathbb{C}_{+}\right)$consists of all functions $f \in H\left(\mathbb{C}_{+}\right)$such that

$$
\|f\|_{p, \alpha}=\left(\int_{0}^{\infty} \int_{-\infty}^{\infty}|f(x+i y)|^{p} y^{\alpha} d x d y\right)^{\frac{1}{p}}<\infty,
$$

where $\alpha>-1$ and $0<p<\infty$ (see [5] and [6]). The above spaces are Banach spaces for $p \geq 1$ and complete metric spaces for $0<p<1$. It is natural to consider the space $A_{\nu}^{\infty}=A_{\nu}^{\infty}\left(\mathbb{C}_{+}\right)$of all holomorphic functions $F$ on $\mathbb{C}_{+}$satisfying

$$
\|F\|_{A_{\nu}^{\infty}}=\sup _{y>0} \sup _{x \in \mathbb{R}}|F(x+i y)| y^{\nu}<\infty,
$$

where $\nu>0$, this space is also a Banach space.

Let $D$ be a bounded strictly pseudoconvex domain in $\mathbb{C}^{n}$ with smooth boundary and let $\delta(z)$ denote the distance from $z \in D$ to the boundary of $D$ with respect to some Riemannian metric, see [1]. We define

$$
L_{s}^{p}(D)=L^{p}\left(D, \delta(z)^{s-\frac{n+1}{p}} d V(z)\right),
$$

where $s p>n, 0<p<\infty$ and $d V(z)$ is the volume element on $D$, (see [1]). Set

$$
\begin{gathered}
A_{s}^{p}(D)=L_{s}^{p}(D) \cap H(D), \quad s p>n, \quad 0<p<\infty, \\
A_{s}^{\infty}(D)=\left\{f \in H(D): \sup _{z \in D}|f(z)| \delta^{s}(z)<\infty\right\}, \quad s \geq 0 .
\end{gathered}
$$

$\dagger$ Supported by Ministry of Science, Serbia, project M144010.

${ }^{1}$ Mathematics Subject Classification 2010 Primary 30D45. Key words and Phrases: Bergman spaces, pseudoconvex domains, extremal problems. 
These spaces are Banach spaces for $1 \leq p \leq \infty$ and complete metric spaces for $0<p<1$.

In this paper we investigate the following two problems: estimate the distance, in $A_{\frac{\nu+2}{q}}^{\infty}\left(\mathbb{C}_{+}\right)$norm, from $f \in A_{\frac{\nu+2}{q}}^{\infty}\left(\mathbb{C}_{+}\right)$to $A_{\nu}^{q}\left(\mathbb{C}_{+}\right)$and estimate the distance, in $A_{s}^{\infty}(D)$ norm, from $f \in A_{s}^{\infty}(D)$ to $A_{s}^{q}$. In both cases we give sharp results. The next section deals with the upper half-plane case, the last one deals with strongly pseudoconvex domains. Techniques used to obtain our results in these two different settings are similar, and the same ideas were used to study analogous problems for analytic Besov spaces in the unit ball and polydisc (see [10] and [11]). The literature on the extremal problems in spaces of analytic functions is extensive, even in the case of the unit disk, a classical exposition of these problems treated by duality methods developed by S. Havinson, W. Rogosinski and H. Shapiro can be found in 8 .

\section{Distance problems in $A_{\frac{\nu+2}{p}}^{p}\left(\mathbb{C}_{+}\right)$SpaCeS}

The main tool in our investigation is Bergman representation formula. We first collect the results needed in the proofs of our theorems. The following result is contained in [5]:

Lemma 1. If $f \in A_{\alpha}^{p}\left(\mathbb{C}_{+}\right), 0<p<\infty$ and $\alpha>-1$ then

$$
f(z)=\frac{\beta+1}{\pi} \int_{0}^{\infty} \int_{-\infty}^{\infty} \frac{f(w)(\Im w)^{\beta}}{(\bar{w}-z)^{2+\beta}} d m_{2}(w),
$$

where $0<p \leq 1, \beta \geq \frac{2+\alpha}{p}-2$ or $1 \leq p<\infty, \beta \geq \frac{1+\alpha}{p}-1$.

Here and in the following $m_{2}$ denotes two dimensional Lebesgue measure. The next two results are contained in [6].

Lemma 2. Let $f \in H\left(\mathbb{C}_{+}\right)$. Assume $\mathbb{C}_{+}$is covered by dyadic squares: $\mathbb{C}_{+}=$ $\cup_{k=1}^{\infty} \Delta_{k}$ and let $\left(\Delta_{k}^{\star}\right)$ be the corresponding family of enlarged squares (see [6]). Then $\Delta_{k}^{\star}$ is a finitely overlapping covering of $\mathbb{C}_{+}$and

$$
\sup _{z \in \Delta_{k}}|f(z)|^{p}(\Im z)^{\alpha} \leq \frac{C}{\left|\Delta_{k}^{\star}\right|} \int_{\Delta_{k}^{\star}}|f(z)|^{p}(\Im w)^{\alpha} d m_{2}(w)
$$

for $0<p<\infty$ and $\alpha>-1$.

Lemma 3. Let $\Delta_{k}$ and $\Delta_{k}^{\star}$ are as in the previous lemma, let $w_{k}$ be the center of the diadic square $\Delta_{k}$. Then we have:

$$
\begin{gathered}
\left(\Im w_{k}\right)^{2} \asymp\left|\Delta_{k}\right|=m_{2}\left(\Delta_{k}\right) \asymp m_{2}\left(\Delta_{k}^{\star}\right), \\
|\bar{w}-z| \asymp\left|\bar{w}_{k}-z\right|, \quad w \in \Delta_{k} \quad, z \in \mathbb{C}_{+}, \\
\Im w \asymp \Im w_{k}, \quad w \in \Delta_{k}
\end{gathered}
$$

and the following integral estimate:

$$
\int_{\mathbb{C}_{+}} \frac{(\Im z)^{\alpha} d m_{2}(z)}{|\bar{w}-z|^{(2+\beta) p}} \leq C(\Im w)^{\alpha+2-(\beta+2) p}, \quad w \in \mathbb{C}_{+}
$$

valid for all $\beta$ satisfying $(\beta+2) p-2>\alpha, \alpha>-1$. 
Theorem 1. (see [3] and [4]) Let $0<p<\infty, \nu>0$. Then there is a constant $C=C(p, \nu)>0$ such that for all $x+i y \in \mathbb{C}_{+}$and all $F \in A_{\nu}^{p}\left(\mathbb{C}_{+}\right)$we have

$$
|F(x+i y)| \leq C y^{-\frac{\nu+2}{p}}\|F\|_{A_{\nu}^{p}\left(\mathbb{C}_{+}\right)} .
$$

Also, there is a constant $C=C(p, \nu)>0$ such that for all $y>0$ and all $F \in A_{\nu}^{p}\left(\mathbb{C}_{+}\right)$ we have

$$
\left(\int_{-\infty}^{\infty}|F(x+i y)|^{p} d x\right)^{1 / p} \leq C y^{-\frac{\nu+1}{p}}\|F\|_{A_{\nu}^{p}\left(\mathbb{C}_{+}\right)} .
$$

We clearly have

$$
\|F\|_{A_{\frac{\nu+2}{p}}^{\infty}} \leq C\|F\|_{A_{\nu}^{p}}
$$

for $0<p<\infty, \nu>0$ and $F \in A_{\nu}^{p}\left(\mathbb{C}_{+}\right)$.

The next two theorems show that $\operatorname{dist}_{A_{\frac{\nu+2}{q}}^{q}}\left(f, A_{\nu}^{q}\right)$ can be explicitly given in the case of the upper halfplane $\mathbb{C}_{+}$. We treat separately cases $0<q \leq 1$ and $q>1$. The distance we are looking for is described using the following sets:

$$
V_{\epsilon, t}(f)=\left\{z=x+i y \in \mathbb{C}_{+}:|f(x+i y)| y^{t} \geq \epsilon\right\} .
$$

Theorem 2. Let $q>1, \nu>-1, t=\frac{\nu+2}{q}, \beta>\max \left(\frac{\nu}{q}, \frac{\nu+2}{q}-1\right)$ and $f \in A_{\frac{\nu+2}{q}}^{\infty}\left(\mathbb{C}_{+}\right)$. Then $l_{1}=l_{2}$ where

$$
\begin{gathered}
l_{1}=\operatorname{dist}_{A_{\frac{\nu+2}{q}}^{\infty}}\left(f, A_{\nu}^{q}\right), \\
l_{2}=\inf \left\{\epsilon>0: \int_{\mathbb{C}_{+}}\left(\int_{V_{\epsilon, t}(f)} \frac{(\Im w)^{\beta-t} d m_{2}(w)}{|\bar{w}-z|^{\beta+2}}\right)^{q}(\Im z)^{\nu} d m_{2}(z)<\infty\right\} .
\end{gathered}
$$

Theorem 3. Let $0<q \leq 1, \nu>-1, t=\frac{\nu+2}{q}, \beta>\frac{\nu+2}{q}-2$ and $f \in A_{\frac{\nu+2}{q}}^{\infty}\left(\mathbb{C}_{+}\right)$. Then $t_{1}=t_{2}$ where

$$
t_{1}=\operatorname{dist}_{\frac{\nu+2}{q}}^{\infty}\left(f, A_{\nu}^{q}\right)
$$

$$
t_{2}=\inf \left\{\epsilon>0: \int_{\mathbb{C}_{+}}\left(\int_{V_{\epsilon, t}(f)} \frac{(\Im w)^{\beta-t} d m_{2}(w)}{|\bar{w}-z|^{\beta+2}}\right)^{q}(\Im z)^{\nu} d m_{2}(z)<\infty\right\} .
$$

We present now the proofs of the above theorems, since they significantly overlap a unified presentation is possible. Assume $l_{1}<l_{2}$ or $t_{1}<t_{2}$. Then there are $\epsilon>\epsilon_{1}>0$ and $f_{\epsilon_{1}} \in A_{\nu}^{q}\left(\mathbb{C}_{+}\right)$such that $\left\|f-f_{\epsilon_{1}}\right\|_{\frac{\nu+2}{q}}^{\infty} \leq \epsilon_{1}$ and

$$
\int_{\mathbb{C}_{+}}\left(\int_{V_{\epsilon, t}(f)} \frac{(\Im w)^{\beta-t} d m_{2}(w)}{|\bar{w}-z|^{\beta+2}}\right)^{q}(\Im z)^{\nu} d m_{2}(z)=+\infty .
$$

Since $\left\|f-f_{\epsilon_{1}}\right\|_{\frac{\nu+2}{q}}^{\infty} \leq \epsilon_{1}$, from the definition of the set $V_{\epsilon, t}(f)$ we conclude that

$$
\left(\epsilon-\epsilon_{1}\right) \chi_{V_{\epsilon, t}(f)}(z)(\Im z)^{-\frac{\nu+2}{q}} \leq\left|f_{\epsilon_{1}}(z)\right|
$$

and therefore we have

$$
+\infty=\int_{\mathbb{C}_{+}}\left(\int_{\mathbb{C}_{+}} \frac{\chi_{V_{\epsilon, t}(f)}(w)(\Im w)^{\beta-t} d m_{2}(w)}{|\bar{w}-z|^{\beta+2}}\right)^{q}(\Im z)^{\nu} d m_{2}(z)
$$




$$
\leq \int_{\mathbb{C}_{+}}\left(\int_{\mathbb{C}_{+}} \frac{\left|f_{\epsilon_{1}}(w)\right|(\Im w)^{\beta}}{|\bar{w}-z|^{2+\beta}} d m_{2}(w)\right)^{q}(\Im z)^{\nu} d m_{2}(z)=M .
$$

Note that this estimate is valid for $0<q<\infty$ and therefore works for both of the above theorems. In both cases we are going to arrive at the contradiction by proving $M<+\infty$. Let us first consider the case $q>1$. Using Hölder's inequality and Lemma 3 (with $\alpha=0$ ) we obtain

$$
\begin{aligned}
I(z) & =\left(\int_{\mathbb{C}_{+}} \frac{\left|f_{\epsilon_{1}}(w)\right|(\Im w)^{\beta}}{|\bar{w}-z|^{2+\beta}} d m_{2}(w)\right)^{q} \\
& \leq \int_{\mathbb{C}_{+}} \frac{\left|f_{\epsilon_{1}}(w)\right|^{q}(\Im w)^{\beta q}}{|\bar{w}-z|^{\beta q-\epsilon q+2}} d m_{2}(w) \cdot\left(\int_{\mathbb{C}_{+}} \frac{d m_{2}(w)}{|\bar{w}-z|^{\epsilon p+2}}\right)^{q / p} \\
& \leq C \int_{\mathbb{C}_{+}} \frac{\left|f_{\epsilon_{1}}(w)\right|^{q}(\Im w)^{\beta q}}{|\bar{w}-z|^{\beta q-\epsilon q+2}} d m_{2}(w)(\Im z)^{-\epsilon q}
\end{aligned}
$$

for $w \in \mathbb{C}_{+}, \epsilon>0$. Using this estimate, Fubini's theorem and Lemma 3 we obtain

$$
\begin{aligned}
M & \leq C \int_{\mathbb{C}_{+}}\left|f_{\epsilon_{1}}(w)\right|^{q}(\Im w)^{\beta q}\left(\int_{\mathbb{C}_{+}} \frac{(\Im z)^{\nu-\epsilon q}}{|\bar{w}-z|^{\beta q-\epsilon q+2}} d m_{2}(z)\right) d m_{2}(w) \\
& \leq C \int_{\mathbb{C}_{+}}\left|f_{\epsilon_{1}}(w)\right|^{q}(\Im w)^{\nu} d m_{2}(w)<\infty
\end{aligned}
$$

where $\epsilon>0$ is small enough so that $\nu-\epsilon q>-1$.

Now let us turn to the case $q \leq 1$. We have, using Lemma 2 and Lemma 3 ,

$$
\begin{aligned}
I(z) & =\left(\int_{\mathbb{C}_{+}} \frac{\left|f_{\epsilon_{1}}(w)\right|(\Im w)^{\beta}}{|\bar{w}-z|^{2+\beta}} d m_{2}(w)\right)^{q} \\
& =\left(\sum_{k} \int_{\Delta_{k}} \frac{\left|f_{\epsilon_{1}}(w)\right|(\Im w)^{\beta}}{|\bar{w}-z|^{2+\beta}} d m_{2}(w)\right)^{q} \\
& \leq C \sum_{k=1}^{\infty} \max _{w \in \Delta_{k}}\left|f_{\epsilon_{1}}(w)\right|^{q}\left(m_{2}\left(\Delta_{k}\right)\right)^{q} \frac{\left(\Im w_{k}\right)^{\beta q}}{|\bar{w}-z|^{(2+\beta) q}} \\
& \leq C \int_{\mathbb{C}_{+}} \frac{\left|f_{\epsilon_{1}}(w)\right|^{q}(\Im w)^{\beta q+2 q-2} d m_{2}(w)}{|\bar{w}-z|^{(2+\beta) q}},
\end{aligned}
$$

in the last estimate we used finite overlapping property of the family of enlarged cubes. Now we get $M<\infty$ as in the case $q>1$, namely by applying Funini's theorem and integral estimate from Lemma 3 .

The reverse inequalities $l_{1} \leq l_{2}$ and $t_{1} \leq t_{2}$ can be proved simultaneously. We fix $\epsilon>0$ such that the integrals in (11), respectively (2), are finite (if there are no such $\epsilon>0$, the inequality is trivial) and use integral representation from Lemma 1. This integral representation is valid for $p=\infty, \alpha>0$ and $\beta$ sufficiently large.

$$
\begin{aligned}
f(z) & =\frac{\beta+1}{\pi}\left(\int_{\mathbb{C}_{+} \backslash V_{\epsilon, t}(f)} \frac{f(w)(\Im w)^{\beta} d m_{2}(w)}{(\bar{w}-z)^{2+\beta}}+\int_{V_{\epsilon, t}(f)} \frac{f(w)(\Im w)^{\beta} d m_{2}(w)}{(\bar{w}-z)^{2+\beta}}\right) \\
& =\frac{\beta+1}{\pi}\left(f_{1}(z)+f_{2}(z)\right)
\end{aligned}
$$


where $\beta$ is sufficiently large. The estimate for $f_{1}(z)$ is immediate, using Lemma 3 .

$$
\left|f_{1}(z)\right| \leq \epsilon \int_{\mathbb{C}_{+}} \frac{(\Im w)^{\beta-t} d m_{2}(w)}{|\bar{w}-z|^{2+\beta}} \leq C \epsilon(\Im z)^{-t}
$$

and this means $f_{1} \in A_{\frac{\nu+2}{q}}^{\infty}$ with $\left\|f_{1}\right\|_{A_{t}^{\infty}}^{\infty} \leq C \epsilon$. Next, by the choice of $\epsilon>0$ we have:

$$
\begin{aligned}
\left\|f_{2}\right\|_{A_{\nu}^{q}} & \leq C\|f\|_{A_{t}^{\infty}} \int_{\mathbb{C}_{+}}\left(\int_{V_{\epsilon, t}(f)} \frac{(\Im w)^{\beta-t} d m_{2}(w)}{|\bar{w}-z|^{2+\beta}}\right)^{q}(\Im z)^{\nu} d m_{2}(z) \\
& \leq C\|f\|_{A_{t}^{\infty}}
\end{aligned}
$$

This completes the proofs of both theorems.

We end this section with an observation that the upper halfplane is the simplest tube domain. Analysis on tube domains over symmetric cones is an active area of research (see [2, 3] and [4]) and availability of integral representations points to possibility of extending our results to this more general setting.

\section{Distance PRoblems in $A_{s}^{q}(\Omega)$ SPACES}

We now consider Bergman type spaces in $D \subset \mathbb{C}^{n}$, where $D$ is smoothly bounded relatively compact strictly pseudoconvex domain, providing also sharp results in this case. Our proofs are heavily based on the estimates from [1], where more general situation was considered.

Since $|f(z)|^{p}$ is subharmonic (even plurisubharmonic) for a holomorphic $f$, we have $A_{s}^{p}(D) \subset A_{t}^{\infty}(D)$ for $0<p<\infty$, sp $>n$ and $t=s$. Also, $A_{s}^{p}(D) \subset A_{s}^{1}(D)$ for $0<p \leq 1$ and $A_{s}^{p}(D) \subset A_{t}^{1}(D)$ for $p>1$ and $t$ sufficiently large. Therefore we have an integral representation

$$
f(z)=\int_{D} f(\xi) K(z, \xi) \delta^{t}(\xi) d V(\xi)
$$

where $K(z, \xi)$ is a kernel of type $n+t+1$, that is a measurable function on $D \times D$ such that $|K(z, \xi)| \leq C|\tilde{\Phi}(z, \xi)|^{-(n+1+t)}$, where $\tilde{\Phi}(z, \xi)$ is so called Henkin-Ramirez function for $D$. From now on we work with a fixed Henkin-Ramirez function $\tilde{\Phi}$ and a fixed kerenel $K$ of type $n+t+1$. We are going to use the following results from [1].

Lemma 4. ([1], Corollary 5.3.) If $r>0,0<p \leq 1, s>-1, p(s+n+1)>n$ and $f \in H(D)$, then we have

$$
\left(\int_{D}|f(\xi)||\tilde{\Phi}(z, \xi)|^{r} \delta^{s}(\xi) d V(\xi)\right)^{p} \leq C \int_{D}|f(\xi)|^{p}|\tilde{\Phi}(z, \xi)|^{r p} \delta^{p(s+n+1)-(n+1)}(\xi) d V(\xi) .
$$

Lemma 5. ([1], Corollary 3.9.) Assume $T(z, \xi)$ is a kernel of type $\beta$, and $\sigma>0$ satisfies $\sigma+n-\beta<0$. Then we have

$$
\int_{D} T(z, \xi) \delta^{\sigma-1}(z) d V(z) \leq C \delta^{\sigma+n-\beta}(\xi) .
$$

A natural problem is to estimate $\operatorname{dist}_{A_{s}^{\infty}(D)}\left(f, A_{s}^{q}(D)\right)$ where $0<q<\infty, s q>n$ and $f \in A_{s}^{\infty}(D)$. We give sharp estimates below, treating cases $0<q \leq 1$ and $q>1$ separately. 
Theorem 4. Let $0<q \leq 1, s q>n, f \in A_{s}^{\infty}(D)$ and $t>s$ is sufficiently large. Then $\omega_{1}=\omega_{2}$ where

$$
\begin{gathered}
\omega_{1}=\operatorname{dist}_{A_{s}^{\infty}(D)}\left(f, A_{s}^{q}(D)\right), \\
\omega_{2}=\inf \left\{\epsilon>0: \int_{D}\left(\int_{\Omega_{\epsilon, s}}|K(z, \xi)| \delta^{t-s}(\xi) d V(\xi)\right)^{q} \delta^{s q-n-1}(z) d V(z)<\infty\right\},
\end{gathered}
$$

where $K(z, \xi)$ is the above kerenel of type $n+t+1$ and

$$
\Omega_{\epsilon, s}=\left\{z \in D:|f(z)| \delta^{s}(z) \geq \epsilon\right\} .
$$

Proof. Let us prove that $\omega_{1} \leq \omega_{2}$. We fix $\epsilon>0$ such that the above integral is finite and use (3):

$$
f(z)=\int_{D \backslash \Omega_{\epsilon, s}} f(\xi) K(z, \xi) d V(\xi)+\int_{\Omega_{\epsilon, s}} f(\xi) K(z, \xi) d V(\xi)=f_{1}(z)+f_{2}(z) .
$$

We estimate $f_{1}$ :

$$
\begin{aligned}
\left|f_{1}(z)\right| & \leq C \epsilon \int_{D}|K(z, \xi)| \delta^{t-s} d V(\xi) \\
& \leq C \epsilon \int_{D} \frac{\delta^{t-s}(\xi) d V(\xi)}{|\tilde{\Phi}(z, \xi)|^{n+t+1}} \\
& \leq C \epsilon \delta^{-s}(z),
\end{aligned}
$$

where the last estimate is contained in [1] (see p. 375). Next,

$$
\begin{aligned}
\left\|f_{2}\right\|_{A_{s}^{q}}^{q} & =\int_{D}\left|f_{2}(z)\right|^{q} \delta^{s q-n-1}(z) d V(z) \\
& \leq C \int_{D}\left(\int_{\Omega_{\epsilon, s}}|f(\xi)| K(z, \xi) \delta^{t}(\xi) d V(\xi)\right)^{q} \delta^{s q-n-1}(z) d V(z) \\
& \leq C^{\prime}\|f\|_{A_{s}^{\infty}}^{q} .
\end{aligned}
$$

Now we have

$$
\operatorname{dist}_{A_{s}^{\infty}(D)}\left(f, A_{s}^{q}(D)\right) \leq\left\|f-f_{2}\right\|_{A_{s}^{\infty}(D)}=\left\|f_{1}\right\|_{A_{s}^{\infty}(D)} \leq C \epsilon .
$$

Now assume that $\omega_{1}<\omega_{2}$. Then there are $\epsilon>\epsilon_{1}>0$ and $f_{\epsilon_{1}} \in A_{s}^{q}(D)$ such that $\left\|f-f_{\epsilon_{1}}\right\|_{A_{s}^{\infty}} \leq \epsilon_{1}$ and

$$
I=\int_{D}\left(\int_{\Omega_{\epsilon, s}}|K(z, \xi)| \delta^{t-s}(\xi) d V(\xi)\right)^{q} \delta^{s q-n-1}(z) d V(z)=\infty .
$$

As in the case of the upper half-plane one uses $\left\|f-f_{\epsilon_{1}}\right\|_{A_{s}^{\infty}} \leq \epsilon_{1}$ to obtain

$$
\left(\epsilon-\epsilon_{1}\right) \chi_{\Omega_{\epsilon, s}}(z) \delta^{-s}(z) \leq C\left|f_{\epsilon_{1}}(z)\right| .
$$

Now the following chain of estimates leads to a contradiction:

$$
\begin{aligned}
I & =\int_{D}\left(\int_{D} \chi_{\Omega_{\epsilon, s}}(\xi) \delta^{t-s}(\xi) K(z, \xi) d V(\xi)\right)^{q} \delta^{s q-n-1}(z) d V(z) \\
& \leq C \int_{D}\left(\int_{D}\left|f_{\epsilon_{1}}(\xi)\right| \delta^{t}(\xi) K(z, \xi) d V(\xi)\right)^{q} \delta^{s q-n-1}(z) d V(z)
\end{aligned}
$$




$$
\begin{aligned}
& \leq C \int_{D}\left(\int_{D}\left|f_{\epsilon_{1}}(\xi)\right| \delta^{t}(\xi) \frac{d V(\xi)}{|\tilde{\Phi}(z, \xi)|^{n+t+1}}\right)^{q} \delta^{s q-n-1}(z) d V(z) \\
& \leq C \int_{D} \int_{D}\left|f_{\epsilon_{1}}(\xi)\right|^{q} \frac{\delta^{s q-n-1}(z) \delta^{q(t+n+1)-(n+1)}(\xi)}{|\tilde{\Phi}(z, \xi)|^{q(n+t+1)}} d V(z) d V(\xi) \\
& \leq C \int_{D}\left|f_{\epsilon_{1}}(\xi)\right|^{q} \delta^{s q-n-1}(\xi) d V(\xi)<\infty
\end{aligned}
$$

where we used Lemma 4 and Lemma 5 with $\beta=q(n+t+1), \sigma=s q-n$.

Next theorem deals with the case $1<q<\infty$.

Theorem 5. Let $q>1, s q>n, t>s, t>\frac{s+n+1}{q}$ and $f \in A_{s}^{\infty}(D)$. Then $\omega_{1}=\omega_{2}$ where

$$
\begin{gathered}
\omega_{1}=\operatorname{dist}_{A_{s}^{\infty}(D)}\left(f, A_{s}^{q}(D)\right), \\
\omega_{2}=\inf \left\{\epsilon>0: \int_{D}\left(\int_{\Omega_{\epsilon, s}}|K(z, \xi)| \delta^{t-s}(\xi) d V(\xi)\right)^{q} \delta^{s q-n-1}(z) d V(z)<\infty\right\} .
\end{gathered}
$$

Proof. An inspection of the proof of the previous theorem shows that it extends to this case also, provided one can prove the estimate:

$$
\begin{aligned}
J & =\int_{D}\left(\int_{D}\left|f_{\epsilon_{1}}(\xi)\right| \delta^{t}(\xi) K(z, \xi) d V(\xi)\right)^{q} \delta^{s q-n-1}(z) d V(z) \\
& \leq C \int_{D}\left|f_{\epsilon_{1}}(\xi)\right|^{q} \delta^{s q-n-1}(\xi) d V(\xi)<\infty
\end{aligned}
$$

where $q>1$. Using Hölder's inequality and Lemma 5 , with $\sigma=1$ and $\beta=n+1+p \epsilon$, we obtain

$$
\begin{aligned}
I(z) & =\left(\int_{D}\left|f_{\epsilon_{1}}(\xi)\right| \delta^{t}(\xi) K(z, \xi) d V(\xi)\right)^{q} \\
& \leq \int_{D} \frac{\left|f_{\epsilon_{1}}(\xi)\right|^{q} \delta^{t q}(\xi) d V(\xi)}{|\tilde{\Phi}(z, \xi)|^{n+1+t q-\epsilon q}} \cdot\left(\int_{D} \frac{d V(\xi)}{|\tilde{\Phi}(z, \xi)|^{n+1+p \epsilon}}\right)^{q / p} \\
& \leq C \int_{D} \frac{\left|f_{\epsilon_{1}}(\xi)\right|^{q} \delta^{t q}(\xi) d V(\xi)}{|\tilde{\Phi}(z, \xi)|^{n+1+t q-\epsilon q}} \delta^{-q \epsilon}(z),
\end{aligned}
$$

and this gives

$$
\begin{aligned}
J & \leq C \int_{D} \int_{D} \frac{\left|f_{\epsilon_{1}}(\xi)\right|^{q} \delta^{t q}(\xi) \delta^{-q \epsilon+s q-n-1}(z)}{|\tilde{\Phi}(z, \xi)|^{n+1+t q-\epsilon q}} d V(z) d V(\xi) \\
& \leq C \int_{D}\left|f_{\epsilon_{1}}(\xi)\right|^{q} \delta^{s q-n-1}(\xi) d V(\xi)<\infty
\end{aligned}
$$

where we again used Lemma 5, with $\beta=n+1+t q-\epsilon q$ and $\sigma=q(s-\epsilon)-n>0$.

Remark. We note that most results of this paper and the previous one ([1]) on distances can be extended to bounded symmetric domains $\Omega \subset \mathbb{C}^{n}$. Indeed, the methods of proofs are based on Bergman representation formula, asymptotic properties of the Bergman kernel and Forelli-Rudin type estimates for integrals

$$
\int_{\Omega} K^{\alpha}(w, w)|K(z, w)|^{\beta} d V(w), \quad \alpha>0, \beta>0 z \in \Omega,
$$


where $K(z, w)$ is a Bergman reproducing kernel for the weighted Bergman space $A_{\alpha}^{2}(\Omega)$. The relevant estimates can be found in [2] and [9].

\section{REFERENCES}

[1] F. Beatrous, Jr. $L^{p}$ estimates for extensions of holomorphic functions, Michigan Math. Jour. 32, (1985) 361-380.

[2] D. Bekolle, C. Berger, L. Coburn and K. Zhu, BMO in the Bergman metric on bounded symmetric domains, Jour. of Func. Analysis, 88 (1990), 319-350.

[3] D. Bekolle and A. Bonami, Estimates for the Bergman and Szego projections in two symmetric domains, Colloq. Math., 68 (1995), 81-100.

[4] D. Bekolle, A. Bonami, G. Garrigos, C. Nana, M. Peloso and F. Ricci Lecture notes on Bergman projections on tube domains over cones: an analytic and geometric viewpoint, preprint 2002.

[5] M. Djrbashian and A. Djrbashian, Integral representations for some classes of analytic functions in the half-plane, DAN SSSR, 285 (1985), 547-550.

[6] A. Djrbashian and K. Karapetyan, Integral inequalities between conjugate pluriharmonic functions in multidimensional domains, Izvestia Acad. Nauk Armenii, (1988), 216-236.

[7] M. Djrbashian and F. Shamoian, Topics in the theory of $A_{\alpha}^{p}$ classes Teubner Texte zur Mathematik, 1988, v 105.

[8] P. Duren, Theory of $H^{p}$ Spaces, Academic Press, 1970.

[9] J. Faraut and A. Koranyi, Function spaces and reproducing kernels on bounded symmetric domains, J. Func. Analysis, 88 (1990), 64-89.

[10] R. Shamoyan, O. Mihić, On new estimates for distances in analytic function spaces in higher dimension, Siberian Electronic Mathematical Reports, 6 (2009), 514-517.

[11] R. Shamoyan, O. Mihić, On new estimates for distances in analytic function spaces in the unit disk, polydisk and unit ball, to appear in Boletin Asociacion Mathematica Venezolana.

Bryansk University, Bryansk Russia

E-mail address: rshamoyan@yahoo.com

Faculty of mathematics, University of Belgrade, Studentski Trg 16, 11000 Belgrade, SERBIA

E-mail address: arsenovic@matf.bg.ac.rs 